\title{
Evaluación del impacto del cambio climático en los indicadores hidrológicos de una cuenca del centro de México con base en un ensamble de modelos climáticos y en el modelo hidrológico SWAT
}

\section{Evaluation of the climate change impact on hydrological indicators over a central Mexican basin based on an ensemble of climate models and SWAT}

\author{
Velázquez-Zapata Juan Alberto \\ Consejo Nacional de Ciencia y Tecnología \\ El Colegio de San Luis, San Luis Potosí \\ Correo: javelazquezza@conacyt.mx \\ Troin Magali \\ École de Technologie Supérieure, Université du Québec \\ Departamento de Ingeniería de la Construcción \\ Correo :Troin.Magali@ouranos.ca
}

\author{
Dávila-Ortiz Rodrigo \\ Universidad Autónoma de San Luis Potosí \\ Facultad de Ingeniería \\ Correo: rodrigo.davort@hotmail.com
}

\section{Resumen}

Este trabajo evalúa la influencia de los Modelos Climáticos Globales (MCG) en la estimación del impacto del cambio climático en los recursos hídricos de una cuenca del centro de México. Las simulaciones climáticas provienen de cinco modelos del proyecto CMIP3 (Coupled Model Intercomparison Project) bajo el escenario de emisión A2. Las simulaciones climáticas se usaron como datos de entrada del modelo hidrológico SWAT (Soil and Water Assessment Tool) para estimar el caudal en el periodo de referencia (1971-2000) y en el periodo futuro (2046-2065). El análisis de los cambios estimados en la cuenca de estudio muestra que todos los MCGs seleccionados proyectan en el futuro un incremento en el promedio anual de la temperatura máxima, con valores entre $2.12^{\circ} \mathrm{C}$ y $3.05^{\circ} \mathrm{C}$. El cambio en la precipitación promedio anual es más variable, con valores entre $-19 \%$ y $+15 \%$. Los resultados de la Señal de Cambio Climático (SCC) en los indicadores hidrológicos muestran diferencias considerables en la magnitud del cambio en el caudal estimado con los cinco MCGs. Por ejemplo, para el horizonte 2050 los MCGs estiman una SCC entre $-30 \%$ y $+4 \%$ para el caudal medio mensual. Los resultados evidencian la influencia del MCG en la variabilidad de la magnitud y la dirección del impacto del cambio climático en los indicadores hidrológicos a nivel cuenca.

Descriptores: MCG, SWAT, impacto del cambio climático, México.

\begin{abstract}
This study assesses the influence of Global Climate Models (GCMs) in the evaluation of climate change impacts on water resources over a central Mexican catchment. The climate simulations came from five CMIP3 (Coupled Model Intercomparison Project) models under the A2 emission scenario. The climate simulations were used to feed the SWAT hydrological model (Soil and Water Assessment Tool) for the reference (1971-2000) and future (2046-2065) periods. The analysis of the projected climate changes over the central Mexican catchment shows that all the GCMs estimate an increase in annual maximum temperature between $2.12^{\circ} \mathrm{C}$ and $3.05^{\circ} \mathrm{C}$ for the $2046-2065$ period. The projected changes in mean annual precipitation are more contrasted, with values between $-19 \%$ and $+15 \%$. The results of the climate change signal on hydrological indicators show considerable differences of the magnitude in streamflow changes between the five GCMs. For example, the projected changes in mean monthly streamflow by the GCMs are expected to vary between $-30 \%$ to $+4 \%$ for the 2050 horizon. Our study emphasises that the GCMs lead to a high level of variability on both the direction and magnitude of the climate change impacts on hydrological indicators at the catchment scale.
\end{abstract}

Keywords: GCMs, SWAT, climate change impact, Mexico. 


\section{INTRODUCCIÓN}

El cambio climático alcanzó gran notoriedad e importancia entre la comunidad científica en las últimas décadas. A nivel global, se observa que el clima ha cambiado y que dicho cambio es mayor que el rango de la variabilidad natural. Existe la hipótesis de que la actividad industrial es el principal motor de este cambio a través de la modificación del balance de energía del planeta. Así, las observaciones en los últimos 150 años muestran que la temperatura promedio mundial se ha elevado en todo el orbe: durante el siglo pasado se produjo en dos fases, de 1910 a $1940\left(0.35^{\circ} \mathrm{C}\right)$, y con mayor fuerza desde 1970 hasta el presente $\left(0.55^{\circ} \mathrm{C}\right)$ (IPCC, 2007). Además del aumento de la temperatura, se han registrado cambios en la precipitación y en la frecuencia de eventos extremos.

Dado que no se conoce con exactitud la magnitud del aumento de la temperatura y las variaciones de la precipitación en el futuro, los cambios en el clima se estiman con base en suposiciones sobre las futuras condiciones sociales y económicas de las sociedades. Esta estimación se basa en diferentes escenarios de emisión de gases de efecto invernadero desarrolladas por el $\mathrm{Pa}$ nel Intergubernamental para el Cambio Climático (IPCC). De esta manera, para la década 2090-2099, el escenario A2 proyecta un aumento de temperatura promedio global de $3.4^{\circ} \mathrm{C}$ (respecto a la década 1980-1990) mientas que el escenario $\mathrm{B} 1$ proyecta el aumento en $1.8^{\circ} \mathrm{C}$.

$\mathrm{El}$ incremento de la temperatura promedio mundial se estima a partir de experimentos realizados con Modelos Climáticos Globales (MCG). Un MCG es una representación matemática del sistema climático a través de un programa informático que simula los procesos físicos de la atmósfera, y por lo tanto, permite evaluar los efectos que diversas concentraciones de gases de efecto invernadero (por ejemplo el dióxido de carbono y el metano) tendrán en el clima global. Los MCGs aproximan los procesos físicos de la atmósfera de manera diferente, por lo que las proyecciones del clima futuro difieren de un modelo a otro. No obstante, todos los MCGs predicen aumento de la temperatura global como consecuencia del aumento de los gases de efecto invernadero (IPCC, 2007).

La evaluación del impacto del cambio climático en el ciclo hidrológico es uno de los mayores retos en la hidrología. En México existen algunos estudios que han evaluado el impacto del cambio climático en los recursos hídricos, por ejemplo, el trabajo de Mendoza y colaboradores (1997) dividió el territorio mexicano en doce zonas hidrológicas para evaluar la vulnerabilidad de diferentes regiones ante el cambio climático en el hori- zonte 2050 con base en diferentes modelos climáticos y escenarios de emisión. Los resultados de este estudio muestran el impacto en la escorrentía, en la humedad del suelo, en la evaporación y en el índice de aridez de distintas zonas del país, donde las cuencas con más probabilidad de afectación son las del río Pánuco, del río Lerma-Santiago y la Península de Baja California. El estudio de Velázquez y colaboradores (2015) evaluó el impacto del cambio climático en una cuenca del centro de México con base en simulaciones de un modelo climático regionalizado y un escenario de emisión. Los resultados estiman una disminución significativa del caudal para el futuro (2041-2070) respecto al periodo histórico (1971-2000).

Estudios realizados en cuencas del Noroeste de México evalúan impactos mixtos con base en el cambio climático. Por ejemplo, el estudio de Tapia y colaboradores (2014) estima que en la cuenca del río Yaqui hay posibilidad de sequía frecuente, alternando con periodos de precipitación alta. Asimismo, el estudio de Robles y colaboradores (2015) evaluó el impacto del cambio climático en la cuenca del río Sonora, y los resultados indican un incremento en la precipitación que podría causar el alza del caudal de entrada a los embalses analizados.

Los resultados de los estudios descritos anteriormente indican que el impacto del cambio climático en los recursos hídricos en México variará de una zona hidrológica a otra, dependiendo de sus características climatológicas, de uso de suelo y de relieve, lo que hace necesaria la evaluación a nivel regional. Los trabajos anteriores se realizaron a partir de uno o dos MCGs.

El objetivo del presente estudio fue evaluar el impacto del cambio climático en indicadores hidrológicos de una cuenca en México a partir de simulaciones de cinco modelos provenientes del experimento CMIP3 (Meehl et al., 2007) con base en el escenario de emisión A2. Las simulaciones climáticas se usaron como datos de entrada al modelo hidrológico SWAT para obtener caudales simulados para un periodo histórico de referencia (1971-2000) y un periodo futuro (2046-2065). Con los caudales simulados se calcularon indicadores hidrológicos mensuales y se evaluó la Señal de Cambio Climático (SCC). El uso de un ensamble de modelos permite evaluar la variabilidad en las proyecciones de la SCC cuando se consideran distintos MCGs.

\section{Desarrollo}

\section{Cuenca de estudio}

La figura 1 muestra la localización, zonas climáticas y corrientes principales de la cuenca del río Tampaón. La 
cuenca tiene un rango de altitud entre $18 \mathrm{~m}$ y $3500 \mathrm{~m}$, una superficie de $23373 \mathrm{~km}^{2}$ y se localiza principalmente en el estado de San Luis Potosí. La cuenca tiene su parteaguas más lejano en el estado de Guanajuato, donde se origina el río Santa María, que corre de este a oeste hasta encontrarse con el río Verde, donde en esta confluencia se toma el nombre de río Tampaón. Otros ríos importantes que aportan considerables volúmenes son el río Gallinas y el río Valles. Finalmente, el río Tampaón confluye con el río Moctezuma para tomar el nombre de río Pánuco, el cual desemboca en el Golfo de México. El cauce principal tiene una longitud de 323.8 km y una pendiente de 0.007 .

La cuenca del río Tampaón presenta una gran variedad de climas debido, a las variaciones de altitud de la Sierra Madre Oriental y, por otro lado, a la influencia marítima del Golfo de México. Así, la cadena montañosa actúa como una barrera orográfica que detiene la humedad atmosférica proveniente del océano, originando tres zonas climáticas muy diferentes: en la parte Oriental predomina el clima cálido y húmedo (Climas tipo A), en la parte central se cuenta con un clima templado semihúmedo (Climas tipo C) y la parte occidental presenta un clima árido y templado (Climas tipo B). Esta variedad de climas origina un gran contraste en la vegetación de la cuenca. Así, se puede encontrar bosque de pino-encino en la zona oriental de la cuenca y matorral con una gran variedad de cactáceas en la zona occidental.

Los datos climatológicos para la cuenca se obtuvieron de la base de datos CLICOM (2016) del Servicio Meteorológico Nacional, periodo 1971-2000, para las estaciones meteorológicas mostradas en la figura 1. La temperatura promedio anual de la cuenca es de $21.5^{\circ} \mathrm{C}$ y la precipitación promedio anual es $1080 \mathrm{~mm}$. Los datos hidrométricos del periodo histórico se recuperaron de la estación "El Pujal" de la Comisión Nacional del Agua (figura 1) a partir de la base de datos Banco Nacional de Aguas (i.e., BANDAS; IMTA, 2016). La figura 2 muestra el ciclo anual de precipitación, temperatura y caudal para la cuenca de estudio.

\section{SiMULACIONES CLIMÁTICAS}

Las simulaciones climáticas provienen de los cinco MCGs mostrados en la tabla 1. La presente investigación se realizó con base en el escenario de emisión A2, que describe un mundo muy heterogéneo y considera un incremento sostenido de la población mundial; además, el crecimiento económico proyectado con el esce-

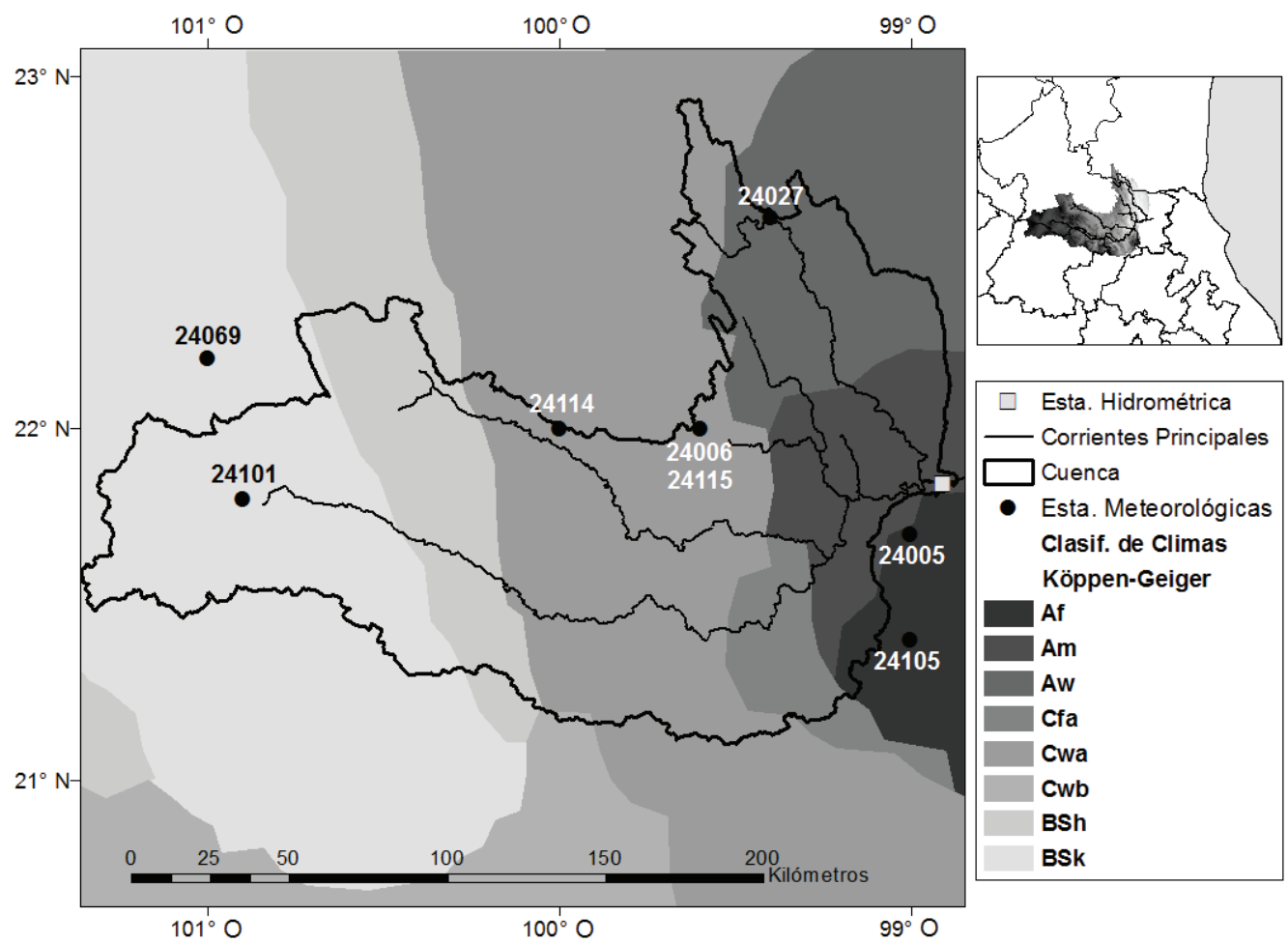

Figura 1. Localización de la cuenca del río Tampaón. Los climas se clasifican en tipo A (tropical), tipo B (árido) y tipo C (templado). Consultar Peel et al. (2007) para detalles de la clasificación de climas KöppenGeiger. El número de la estación meteorológica corresponde al asignado en la base de datos CLICOM (2016) 
nario A2 es regional y el cambio tecnológico hacia fuentes de combustible más limpias es más lento que en otras familias de escenarios (IPCC, 2007). Por lo tanto, este escenario estima un aumento de temperatura medio global mayor que el resto de los escenarios de emisión, lo cual generaría a su vez un impacto mayor en el régimen hidrológico de la cuenca de estudio. Por otro lado, el estudio de Hawkins and Sutton (2009) que evalúa el cambio en la temperatura media global, revela que en los próximos años y hasta el 2025, los diferentes modelos y escenarios estiman cambios muy semejantes, demostrando el efecto retardado de las emisiones futuras; por lo tanto, este estudio se enfoca entre el periodo de 2046 a 2065 en donde la variabilidad de las proyecciones que aporta el modelo climático es más importante.

Las simulaciones climáticas no se emplean directamente como datos de entrada en los modelos hidrológicos, debido al sesgo sistemático (i.e. bias) que las simulaciones tienen respecto a las observaciones (Teutschbein y Seibert, 2012). Por otro lado, los MCGs tienen una escala muy grande que no es adecuada para la simulación hidrológica. Los métodos estadísticos de corrección de sesgo (i.e. bias correction) permiten corregir el error sistemático entre observaciones y simulaciones climáticas. El método seleccionado para este estudio es el Daily Translation (DT); (Mpelasoka y Chiew, 2009), que encuentra una función estadística entre las simulaciones climáticas y las observaciones en el periodo histórico. Dado que la función se aplica a la serie de observaciones de cada estación meteorológica, las simulaciones provenientes de los MCG se regionalizan al mismo tiempo que se corrige el sesgo.

La corrección de sesgo se realiza como describen las ecuaciones siguientes

$$
\begin{aligned}
& T_{\operatorname{corr}(d)}^{r e f}=T_{s i m(d)}^{r e f}+\left(T_{o b s(m, q)}^{r e f}-T_{\operatorname{sim}(m, q)}^{r e f}\right) \\
& P_{\operatorname{corr}(d)}^{r e f}=P_{\operatorname{sim}(d)}^{r e f}\left(\frac{P_{o b s(m, q)}^{r e f}}{P_{\operatorname{sim}(m, q)}^{r e f}}\right)
\end{aligned}
$$
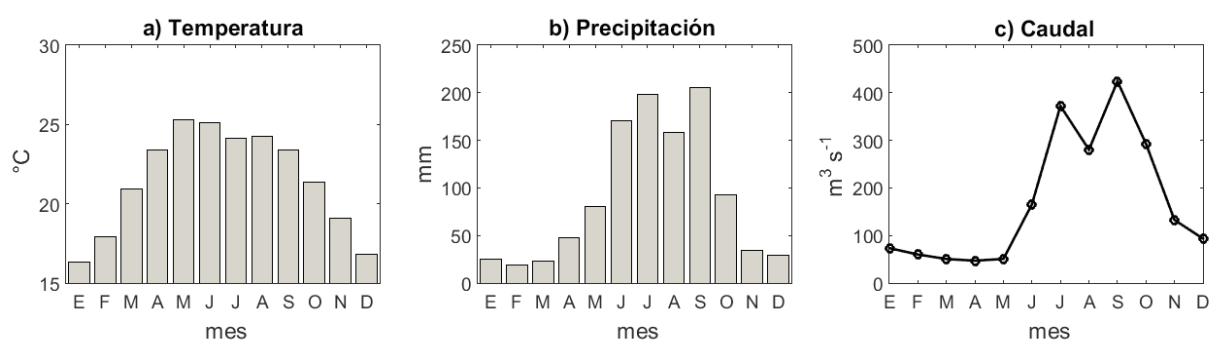

Figura 2. Temperatura, precipitación y caudal promedio observados en la cuenca de estudio (1971-2000) donde

$T_{\text {corr }}$ y $P_{\text {corr }}=$ temperatura y precipitación corregidas

$(q)=$ percentil

(d) $=$ paso de tiempo diario

$(m)=$ mes

$(\operatorname{sim})=$ simulaciones sin corregir

$(o b s)=$ observaciones

En este estudio, la corrección de sesgo se realizó para 50 percentiles. A continuación, la función estadística encontrada en el periodo de referencia se aplica a las simulaciones diarias de clima futuro bajo la hipótesis de que la relación entre simulaciones y observaciones en el futuro será la misma que en periodo histórico (Ho et al., 2012). Para el periodo futuro (fut), las variables corregidas se obtienen con

$$
\begin{aligned}
& T_{\operatorname{corr}(d)}^{\text {fut }}=T_{\operatorname{sim}(d)}^{f u t}+\left(T_{o b s(m, q)}^{r e f}-T_{\operatorname{sim}(m, q)}^{r e f}\right) \\
& P_{\operatorname{corr}(d)}^{f u t}=P_{\operatorname{sim}(d)}^{f u t}\left(\frac{P_{o b s}^{r e f}(m . q)}{P_{\operatorname{sim}(m, q)}^{r e f}}\right)
\end{aligned}
$$

El método DT se ha utilizado ampliamente en estudios de impacto del cambio climático en recursos hídricos (e.g. Teutschbein and Seibert, 2012; Chen et al., 2013; Levison et al., 2014).

El balance hídrico de la cuenca se estimó con el modelo hidrológico SWAT (Soil and Water Assessment Tool (Arnold et al., 1998). Los datos de entrada de SWAT son las temperaturas diarias máximas y mínimas, así como la precipitación promedio diaria. SWAT es un modelo semidistribuido, esto es, toma en cuenta la variabilidad espacial, del uso y tipo de suelo en la estimación de caudales al dividir la cuenca en múltiples unidades de respuesta hidrológica (URH). El cálculo del balance hídrico de la cuenca se estima con la siguiente ecuación

$W_{t}=W_{O}+\sum_{i=1}^{t}\left(P_{i}-Q_{i s u p}-E T_{i}-w_{i}-Q_{\text {isub }}\right)$

donde 
$W_{t} \quad=$ contenido de agua para el tiempo $t$

$W_{o}$ = contenido de agua inicial

$P_{i} \quad=$ precipitación en el día $i$

$Q_{\text {isup }}=$ escurrimiento superficial en el día $i$

$E T_{i}=$ evapotranspiración en el día $i$

$w_{i} \quad=$ agua infiltrada en el suelo en el día $i$

$Q_{i s u b}=$ flujo que proviene del agua subterránea en el día $i$

Los datos de relieve utilizados en SWAT corresponden al Continuo de Elevaciones Mexicano 3.0 (INEGI, 2013).

\section{DISEÑO EXPERIMENTAL}

La cadena de modelación para evaluar el impacto del cambio climático a nivel cuenca incluye la calibración y validación del modelo hidrológico en el periodo histórico, y el uso de simulaciones climáticas como datos de entrada al modelo para obtener series de caudales tanto en el periodo de referencia como en el futuro. A continuación se calculan indicadores hidrológicos y la señal de cambio climático se evalúa (e.g. Muerth et al., 2013; Seiller and Anctil, 2014; Troin et al., 2015; Velázquez et al., 2015).

En el estudio, SWAT se calibró para el periodo 19711985 y se validó durante el periodo 1986-2000. El coeficiente Nash-Sutcliffe (NS; Nash and Sutcliffe, 1970) se utilizó para evaluar la eficiencia del modelo en la simulación de caudales diarios. Un valor de NS $=1$ representa la correspondencia perfecta entre observaciones y simulaciones. Se obtuvieron valores de NS entre 0.91 y 0.85 para los periodos de calibración y validación, respectivamente. La tabla 2 muestra los parámetros más sensibles en la calibración de SWAT para la cuenca de estudio y su descripción.

En el segundo paso, las simulaciones climáticas (una vez corregido el sesgo) se emplearon como datos de entrada en SWAT para simular el caudal tanto en el periodo histórico (1971-2000) como en el periodo futuro (2046-2065). Finalmente se estimó la Señal de Cambio Climático (SCC) en los indicadores hidrológicos calculados con las simulaciones hidrológicas $\left(\mathrm{IH}_{\text {sim }}\right)$.
Los indicadores hidrológicos seleccionados son:

- Caudal promedio mensual $\left(\mathrm{Q}_{\mathrm{m}}\right)$ : es el promedio calculado con todos los valores diarios para un mes dado.

- Caudal promedio máximo mensual $\left(\mathrm{Q}_{\max }\right)$ : es el promedio de los caudales máximos para un mes dado.

- Caudal promedio mínimo mensual $\left(\mathrm{Q}_{\min }\right)$ : es el promedio de los caudales mínimos para un mes dado.

La SCC se calculó con la siguiente ecuación

$$
S C C=\frac{I H_{\text {sim }}^{f u t}-I H_{\text {sim }}^{r e f}}{I H_{\text {sim }}^{r e f}}
$$

\section{ResUltados y DisCUSIÓN}

\section{CAMBIOS PROYECTADOS EN TEMPERATURA Y PRECIPITACIÓN}

La figura 3 muestra el cambio en el promedio anual para la temperatura máxima $\left(\mathrm{T}_{\max }\right)$, la temperatura mínima $\left(\mathrm{T}_{\min }\right)$ y la precipitación $(\mathrm{P})$ entre el periodo histórico y el periodo futuro. El cambio más importante en $\mathrm{T}_{\max }$ se estima para el periodo de primavera, con valores entre $2.3^{\circ}$ y $3.8^{\circ}$, mientras que el invierno es la estación donde se estima el menor incremento, con valores entre $1.5^{\circ} \mathrm{C}$ y $2.6^{\circ}$ (figura 3a). Como puede notarse en esta figura, los valores del cambio en las variables meteorológicas varían según el MCG considerado. Por ejemplo, el mayor cambio en $\mathrm{T}_{\max }$ para verano y otoño se proyecta por CGC, mientras que el menor se proyecta por CM3 (figura 3a). Por otro lado, el cambio estimado en $\mathrm{T}_{\min }$ es menor que el cambio en $\mathrm{T}_{\max }$. Así, el cambio $\mathrm{T}_{\min }$ en primavera varía entre $1.2^{\circ} \mathrm{C}$ y $2.7^{\circ} \mathrm{C}$, donde los modelos MK5 y MRI son los que generalmente presentan el mayor y el menor cambio de $\mathrm{T}_{\text {min, }}$, respectivamente (figura $3 b)$. Los resultados varian más para la precipitación, donde se estiman cambios tanto positivos como negativos dependiendo del MCG considerado. Por ejemplo, para el periodo de lluvias (verano) se evalúa un cambio entre $-20 \%$ y $+9 \%$. Estos resultados muestran la variabilidad que existe en las simulaciones climáticas prove-

Tabla 1. Modelos climáticos globales considerados en este estudio

\begin{tabular}{llc}
\hline \multicolumn{1}{c}{ Instituto } & Identificador CMIP3 & Identificador del estudio \\
\hline $\begin{array}{l}\text { Canadian Centre for Climate Modelling \& Analysis } \\
\text { (Canada) }\end{array}$ & CGCM3.1 & CGC \\
$\begin{array}{l}\text { Météo-France (Francia) } \\
\text { Meteorological Institute of the University of Bonn, } \\
\text { (Alemania) }\end{array}$ & CNRM-CM3 & CM3 \\
CSIRO Atmospheric Research (Australia) & ECHO-G & ECH \\
Meteorological Research Institute (Japón) & CSIRO-Mk3.5 & MK5 \\
\hline
\end{tabular}


Tabla 2. Parámetros más sensibles en la calibración de SWAT, su rango y los valores ajustados para la cuenca de estudio

\begin{tabular}{|c|c|c|c|c|}
\hline Proceso & Parámetro & Descripción & Rango & Valor ajustado \\
\hline Infiltración & $\mathrm{CN} 2$ & Valor inicial de SCS CN2 & $\pm 25 \%$ & -13.8 \\
\hline Evaporación & ESCO & $\begin{array}{l}\text { Factor de compensación } \\
\text { por la evaporación del } \\
\text { suelo }\end{array}$ & $0-1$ & 0.7 \\
\hline Agua subterránea & GWQMN & $\begin{array}{l}\text { Profundidad del umbral } \\
\text { de agua en el acuífero } \\
\text { superficial requerido por } \\
\text { el flujo base }\end{array}$ & $\begin{array}{l}0-1000 \\
\mathrm{~mm}\end{array}$ & 686 \\
\hline $\begin{array}{l}\text { Escurrimiento } \\
\text { superficial }\end{array}$ & SURLAG & $\begin{array}{l}\text { Coeficiente de retardo del } \\
\text { escurrimiento superficial }\end{array}$ & 1-24 días & 10 \\
\hline Intercepción & CANMX & $\begin{array}{l}\text { Máxima intercepción por } \\
\text { follaje }\end{array}$ & $0-10 \mathrm{~mm}$ & 8.9 \\
\hline Cultivo & BLAI & $\begin{array}{l}\text { Índice máximo potencial } \\
\text { de área foliar para cultivos }\end{array}$ & $0-1$ & 0.98 \\
\hline Suelo & SOL_AWC & $\begin{array}{l}\text { Capacidad de agua } \\
\text { disponible para las plantas }\end{array}$ & $\pm 25 \%$ & 24.7 \\
\hline
\end{tabular}

nientes de diferentes MCG y que, como se verá más adelante, se transmite a las simulaciones hidrológicas. En cuanto a promedios anuales, el rango del cambio en temperatura máxima varía entre $2.12^{\circ} \mathrm{C}(\mathrm{MRI})$ y $3.05^{\circ} \mathrm{C}$ (CGC) y para la precipitación anual el rango varía entre $-19 \%$ (CGC) y $+15 \%$ (CM3).

La evaluación del posible cambio de la temperatura en la zona de estudio muestra que las estimaciones de los MCGs coinciden con un aumento en la temperatura para el futuro. Sin embargo, las estimaciones del cambio en la precipitación en la región son más variadas. La causa de estas diferencias radica en las diferentes conceptualizaciones de algunos procesos que regulan la precipitación y que no pueden representarse de manera explícita en los modelos climáticos (e.g., la nubosidad) y por lo tanto, deben incluirse de manera aproximada. Además existen limitaciones en cuanto al conocimiento científico y a la disponibilidad de observaciones detalladas de algunos procesos físicos. En general, la confianza que se tiene en las estimaciones de los modelos climáticos es mayor para algunas variables climáticas, por ejemplo, la temperatura, que para otras, como la precipitación (IPCC, 2007).

\section{CAmbios en el RÉGIMEN HIDROLÓGICO}

La figura 4a muestra los hidrogramas simulados por SWAT con las simulaciones climáticas en el periodo de referencia (1971-2000), las cuales reproducen correctamente el caudal simulado con observaciones. Se analiza que en el periodo de referencia existen dos extremos mensuales, en julio y septiembre, y que el ciclo anual se representa correctamente por SWAT a partir de las simulaciones de los MCGs (figura 1). El caudal del río Tampaón disminuye en agosto debido a la sequía interestival que se presenta en el ciclo de precipitación del centro y sur de México (Magaña et al., 1999).

Los promedios mensuales de caudal estimados con SWAT para el periodo futuro (2046-2065) se muestran en la figura $4 \mathrm{~b}$. Se observan cambios de caudales tanto positivos como negativos, dependiendo del MCG considerado. Por ejemplo, CGC estima un cambio negativo en el futuro, respecto al periodo de referencia, mientras que ECH y CM3 proyectan un cambio de caudal positivo para el extremo de septiembre. Asimismo, estos tres MCG estiman que el ciclo anual que se presenta en el periodo de referencia se conservará en el futuro. Por otro lado, MK5 y MRI simulan un cambio en el ciclo anual en el futuro, dado que el extremo de julio es ma- 

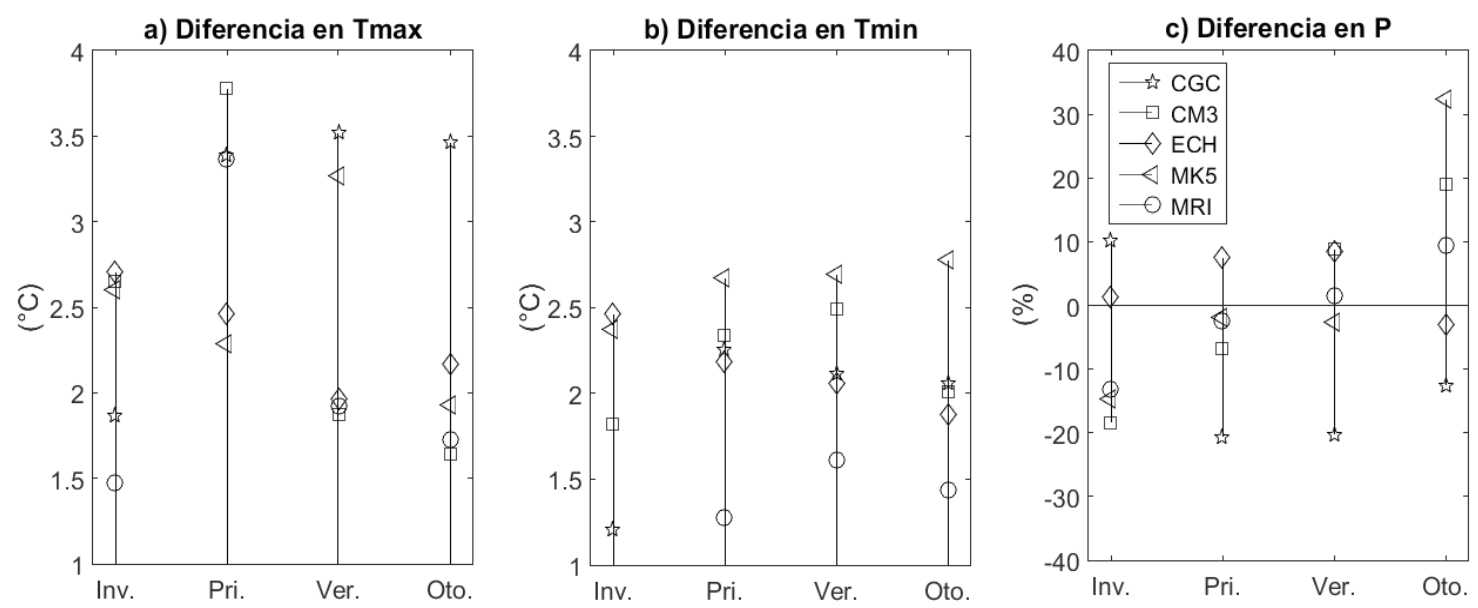

Figura 3. Cambio en el promedio de temperatura máxima $\left(T_{\max }\right)$, temperatura mínima $\left(T_{\min }\right)$ y precipitación $(P)$ entre el periodo futuro (2046-2065) y el periodo histórico (1971-2000) para el invierno (Inv.), la primavera (Pri.), el verano (Ver.) y el otoño (Oto.) calculados para la cuenca de estudio

yor que el de septiembre, mientras que ECH no estima el periodo de sequía interestival.

La figura 5 muestra la Señal de Cambio Climático (SCC) obtenida para los tres indicadores hidrológicos $\left(\mathrm{Q}_{\mathrm{m}^{\prime}} \mathrm{Q}_{\min } \mathrm{y} \mathrm{Q}_{\max }\right)$. Cada diagrama de caja muestra los 12 valores mensuales para un indicador y un modelo dado. Podemos notar que CGC proyecta un decremento en los tres indicadores, ya que el valor mediano es de $-30 \%,-28$ y $-32 \%$ para $Q_{m^{\prime}} Q_{\min }$ y $Q_{\max ,}$ respectivamente, donde es el único MCG que tiene estimaciones negativas en todo su rango. El resto de los MCGs proyecta cambios tanto positivos como negativos para los indicadores seleccionados, donde los valores medianos positivos son para CM3, ECH y MRI y negativos para
MK5. Sin embargo, el valor mediano de la SCC es de magnitud comparable, por ejemplo, para $Q_{m}$ la mediana de la SCC varía entre $+4 \%$ (para CM3) y $-7 \%$ (para MK5, figura 5a). Si bien la mediana de la SCC puede mostrar un incremento o decremento, existen casos en que el rango de SCC de un modelo cubre el rango de la SCC de otro modelo. Por ejemplo, el rango de MK5 para $Q_{\min }$ (entre +25 y $-25 \%$ ) cubre el rango de CM3 (+22 y $-23 \%)$, si bien el primer modelo tiene una SCC mediana negativa y el segundo una SCC mediana positiva (figura $5 b$ ).

La prueba de Wilcoxon (Wilcoxon 1945) se usó para comparar la SCC obtenida con dos diferentes GCMs (mostradas en la figura 5). La hipótesis nula es que dos
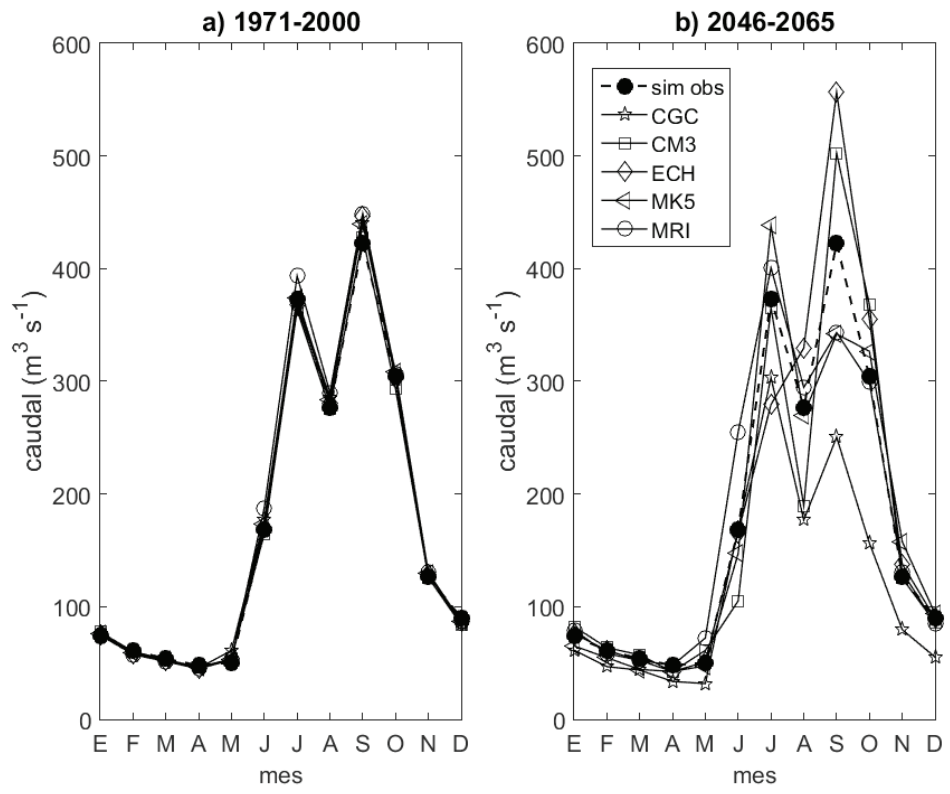

Figura 4. Hidrogramas para la cuenca de estudio simulados en a) el periodo histórico y b) en el periodo futuro. Se muestra en las dos secciones de la figura la simulación de SWAT con las observaciones en el periodo 1971-2000 (sim obs) 
series de datos son muestras independientes de la misma distribución continua, contra la hipótesis alternativa de que no lo son (Wilks, 2006). La tabla 3 muestra el valor $p$ (del inglés $p$-value) obtenido con la prueba de Wilcoxon para los pares de modelos, donde $5 \%$ es el valor de rechazo de la hipótesis nula.

Los resultados de la tabla 3 muestran que la hipótesis nula se rechazó para todos los casos en que se comparó CGC con algún otro modelo. En la prueba al resto de pares de modelos, la hipótesis nula no se rechazó. Puede observarse que, de acuerdo con la prueba estadística, con cuatro de cinco modelos utilizados se obtienen SCC comparables en magnitud y por lo tanto, pueden usarse con cierta confianza para comunicar el rango de la SCC obtenida. Así, la SCC mediana proyectada se encuentra entre $+4 \%$ y $-7 \%$, para $Q_{m^{\prime}}$ entre +4 y $-5 \%$ para $Q_{\min } y$ entre +6 y $-10 \%$ para $Q_{\max }$. En otras palabras, la magnitud de la SCC proyectada es comparable para cuatro de los modelos seleccionados, aunque la dirección varía (hay incrementos y decrementos).

La evaluación del riesgo asociado al cambio climático se establece en relación con el grado de confianza que se tiene de que el riesgo se presentará. Así, se tiene un nivel de confianza alto de que los cambios en la temperatura y la precipitación en el futuro tendrán un efecto en el ciclo hidrológico en todo el orbe (Arnell y Gosling, 2013). Sin embargo, el impacto en los recursos hídricos no depende exclusivamente del clima, sino también está ligado a cambios en el uso de suelo (como consecuencia de las actividades de la industria agropecuaria y forestal), a la gestión de los recursos hídricos, y a las condiciones sociales, económicas y políticas de las regiones posiblemente afectadas (Conde, 2010).

La evaluación del impacto del cambio climático en los recursos hídricos se afecta por los múltiples pasos que comprende la cadena de simulación hidrológica (MCG, el escenario de emisión, el método de corrección de sesgo y el modelo hidrológico). La presente investigación evaluó la señal de cambio climático en los indicadores hidrológicos para una cuenca de México con simulaciones provenientes de cinco modelos climáticos del experimento CMIP3, los cuales se consideran igualmente probables para la evaluación del impacto del cambio climático (IPCC, 2007). Estudios en la literatura muestran que la elección del MCG es una fuente importante de incertidumbre (e.g. Graham et al., 2007). Los resultados muestran que con cuatro GCMs se obtienen SCC comparables en magnitud (aunque varían en sentido). El modelo restante (CGC) proyecta un cambio negativo importante y sobresale entre la muestra de MCGs.

Por otra parte, trabajos previos señalan que la variabilidad en la señal del impacto del cambio climático asociada a los modelos hidrológicos es significativamente menor a la que proviene de los MCGs (e.g. Di-
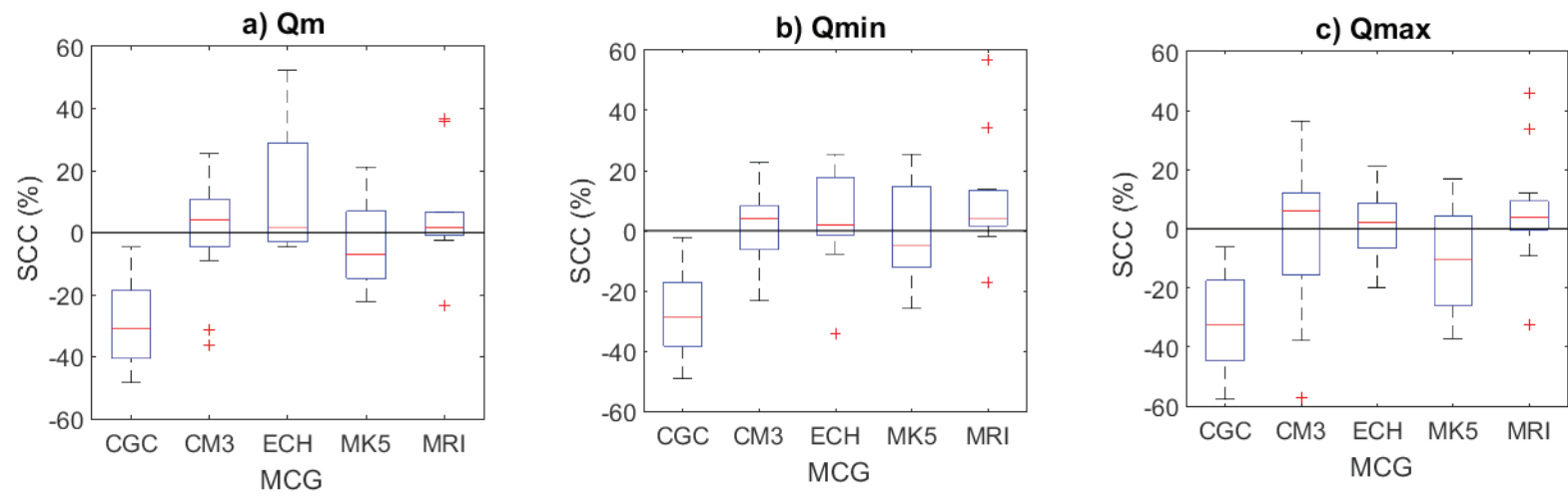

Figura 5. Señal de cambio climático (SCC) en los indicadores hidrológicos. Cada diagrama de caja toma en cuenta los doce valores mensuales obtenidos para un indicador y un MCG dado. En los diagramas de caja la línea central es la mediana y los límites de la caja son los percentiles 25 y 75

Tabla 3. Resultados de la prueba de Wilcoxon realizados a los pares de series de la SCC de indicadores hidrológicos (figura 5). Se muestra el valor $\mathrm{p}$ (del inglés, p-value). En esta prueba, la hipótesis nula se rechazó al nivel de 5\%

\begin{tabular}{lcccccccccc}
\hline & CGC-CM3 & CGC-ECH & CGC-MK5 & CGC-MRI & CM3-ECH & CM3-MK5 & CM3-MRI & ECH-MK5 & ECH-MRI & MK5-MRI \\
\hline $\mathrm{Q}_{\mathrm{m}}$ & $<0.001$ & $<0.001$ & $<0.001$ & $<0.001$ & 0.840 & 0.341 & 0.977 & 0.089 & 0.977 & 0.141 \\
$\mathrm{Q}_{\min }$ & $<0.001$ & $<0.001$ & $<0.001$ & $<0.001$ & 0.885 & 0.471 & 0.583 & 0.194 & 0.471 & 0.141 \\
$\mathrm{Q}_{\max }$ & $<0.001$ & $<0.001$ & $<0.001$ & $<0.001$ & 0.751 & 0.312 & 0.990 & 0.100 & 0.624 & 0.061 \\
\hline
\end{tabular}


bike and Coulibaly, 2005; Crosbie et al., 2011). Sin embargo, el estudio de Velázquez et al. (2013) muestra que la selección del modelo hidrológico tiene una influencia importante cuando se estima el impacto del cambio climático en los caudales pequeños durante la época seca. Por lo tanto, es necesario que el trabajo futuro incluya un ensamble de otros modelos hidrológicos para evaluar la respuesta hidrológica de la cuenca a la estructura del modelo, con lo que aumentará nuestra confianza en las proyecciones del impacto del cambio climático en los indicadores hidrológicos. Asimismo, el trabajo futuro en cuencas mexicanas deberá incluir los recientes escenarios de emisión del IPCC (IPCC, 2014).

\section{COnClusiones}

Los estudios de impacto del cambio climático realizados previamente en cuencas mexicanas han considerado solo uno o dos MCGs, lo que puede limitar la percepción de la Señal de Cambio Climático (SCC). La excepción es el trabajo de Rivas y colaboradores (2010) que combinó simulaciones climáticas provenientes de 23 MCGs para estimar el impacto del cambio climático en tres grandes cuencas de México. Sin embargo, Arnell y Gosling (2013) consideran que el promediar cambios positivos y negativos en las variables meteorológicas, antes de realizar las simulaciones hidrológicas, puede llevar a una pérdida de la SCC en los indicadores hidrológicos.

Con el fin de evaluar la variabilidad de la SCC asociada al modelo climático en la estimación del caudal futuro del río Tampaón, la presente investigación utilizó las simulaciones de un ensamble de cinco MCGs que provienen del experimento CMIP3. Para ello, las simulaciones climáticas se usaron para alimentar el modelo hidrológico SWAT en un periodo histórico de referencia (1971-2000) y en un periodo futuro (2041-2065).

Los resultados de Rivas et al. (2010), al combinar simulaciones climáticas de diversos MCGs, estiman una disminución de la precipitación y en el escurrimiento medio anual en el futuro. En contraste, los resultados de nuestra investigación muestran que los MCGs considerados estiman diferentes cambios en la precipitación promedio anual, con valores tanto positivos como negativos. Como resultado, la SCC en los indicadores hidrológicos mensuales varía según el modelo climático. Así, cuatro modelos proyectan una SCC similar en magnitud aunque de sentido diferente (tanto positivo como negativo). Por otro lado, un modelo (CGC) sobresale al proyectar una SCC fuertemente negativa en los tres indicadores. El estudio de Velázquez et al., (2015) utilizó únicamente este último modelo climático (regio- nalizado dinámicamente) para estimar el impacto del cambio climático en indicadores hidrológicos en una cuenca de México y sus resultados proyectan un decremento importante en los indicadores hidrológicos seleccionados. El uso de un solo MCG, o de la combinación de diversos MCGs puede dar una estimación incompleta del probable impacto del cambio climático en los recursos hídricos. Por lo tanto, nuestros resultados muestran la necesidad de considerar un ensamble de modelos climáticos para tomar en cuenta la variabilidad en las proyecciones de precipitación y temperatura, y que a su vez, se transmite a las simulaciones hidrológicas.

\section{AgradeCimientos}

Agradecemos a los grupos de investigación del Programa de Diagnóstico e Intercomparación de Modelos Climáticos (PCMDI) y al WCRP's Grupo de Trabajo sobre Modelado Acoplado (WGCM) por hacer disponibles los datos de los modelos climáticos. Asimismo, los autores agradecen a los tres dictaminadores anónimos por sus comentarios y sugerencias. Rodrigo Dávila agradece la beca de investigación otorgada por El Colegio de San Luis A.C.

\section{RefERENCIAS}

Arnell N.G. and Gosling S.N. The impacts of climate change on river flow regimes at the global scale. Journal of Hydrology, volume 486, 2013: 351-364.

Arnold J.G., Srinivasan R., Muttiah R.S., Williams J.R. Large area hydrologic modelling and assessment-Part I: model development. Journal of the American Water Resources Association, volume 34, 1998: 73-89.

Chen J., Brissette F.P., Chaumont D., Braun M. Performance and uncertainty evaluation of empirical downscaling methods in quantifying the climate change impacts on hydrology over two North American river basins. Journal of Hydrology, volumen 479, 2013: 200-214.

CLICOM. Base de Datos Climatológica Nacional [en línea] [fecha de consulta: 8 de noviembre de 2016], Centro de Investigación Científica y de Educación Superior de Ensenada, Ensenada, México, 2016. Disponible en: http://clicom-mex.cicese.mx.

Conde A.C. El cambio climático. De lo inequívoco a lo incierto, en: Delgado G.C., Gay C., Martinez M.A, México frente al cambio climático. Retos y oportunidades, $1^{\text {a }}$ ed., México, UNAM, 2010, pp. 17-33.

Crosbie R.S., Dawes W.R., Charles S.P., Mpelasoka F.S., Aryal S., Barron O., Summerell G.K. Differences in future recharge estimates due to GCMs, downscaling methods and hydrological models. Geophysical Research Letters, volumen 38 (número 11), L11406, 2011. doi:10.1029/2011g1047657. 
Dibike Y.B. y Coulibaly P. Hydrologic impact of climate change in the Saguenay watershed: comparison of downscaling methods and hydrologic models. Journal of Hydrology, volumen 307 (número 1), 2005: 145-163. doi:10.1016/j.jhydrol.2004.10.012.

Graham L.P., Hagemann S., Jaun S., Beniston M. On interpreting hydrological change from regional climate models. Climatic Change, volumen 81 (número 1), 2007: 97-122.

Hawkins E. y Sutton R. The potential to narrow uncertainty in regional climate predictions. Bulletin of the American Meteorological Society, volumen 90 (número 8), 2009: 1095-1107.

Ho C.K., Stephenson D.B., Collins M., Ferro C.A.T., Brown S.J. Calibration strategies: a source of additional uncertainty in climate change projections. Bulletin of the American Meteorological Society, volumen 93 (número 1), 2012: 21-26.

INEGI. Continuo de Elevaciones Mexicano 3.0 (CEM 3.0) [en línea] [fecha de consulta: 16 de febrero de 2017], Instituto Nacional de Estadística Geografía e Informática, Aguascalientes, México, 2013. Disponible en: http://www.inegi.org.mx/geo/contenidos/ datosrelieve/continental/continuoelevaciones.aspx

IMTA, Banco Nacional de Datos de Aguas Superficiales (BANDAS) [en línea] [fecha de consulta: 8 de noviembre de 2016], Instituto Mexicano de Tecnología del Agua, Jiutepec, Morelos, México, 2016. Disponible en: http://www.conagua.gob.mx/ CONAGUA07/Contenido/Documentos/Portada\%20BANDAS.htm

IPCC. Contribution of Working Group I to the Fourth Assessment Report of the Intergovernmental Panel on Climate Change, en: Solomon S., Qin D., Manning M., Chen Z., Marquis M., Averyt K.B., Tignor M., Miller H.L. (eds.), Cambridge University Press, Cambridge, United Kingdom and New York, 2007, 996 p.

IPCC. Summary for policymakers. Climate Change 2014: Impacts, Adaptation, and Vulnerability. Part A: Global and Sectoral Aspects. Contribution of Working Group II to the Fifth Assessment Report of the Intergovernmental Panel on Climate Change en: Field C.B., Barros V.R., Dokken D.J., Mach K.J., Mastrandrea M.D., Bilir T.E., Chatterjee M., Ebi K.L., Estrada Y.O., Genova R.C., Girma B., Kissel E.S., Levy A.N., MacCracken S., Mastrandrea P.R., White L.L. (eds.), Cambridge University Press, Cambridge, United Kingdom and New York, 2014, 1132 p.

Levison J., Larocque M., Fournier V., Gagné S., Pellerin S., Ouellet M.A. Dynamics of a headwater system and peatland under current conditions and with climate change. Hydrological Processes, volumen 28 (número 17), 2014: 4808-482. doi: 10.1002/ hyp.9978.

Magaña V., Amador J.A., Medina S. The midsummer drought over Mexico and Central America. Journal of Climate, volumen 12 (número 6), 1999: 1577-1588.

Meehl G.A., Covey C., Taylor K.E., Delworth T., Stouffer R.J., Latif M., McAvaney B., Mitchell J.F.B. THE WCRP CMIP3 Multimodel Dataset: A New Era in Climate Change Research. Bulle- tin of the American Meteorological Societ., volumen 88 (número 9), 2007: 1383-1394.

Mendoza V.M., Villanueva E.E., Adem J. Vulnerability of basins and watersheds in Mexico to global change. Climate Research, volume, 9 (números 1-2), 1997: 139-145.

Mpelasoka F.S. y Chiew F.H.S. Influence of rainfall scenario construction methods on runoff projections, Journal of Hydrometeorology, volumen 10 (número 5), 2009: 1168-1183.

Muerth M.J., Gauvin-St-Denis B., Ricard S., Velázquez J.A., Schmid J., Minville M., Caya D., Chaumont D., Ludwig R., Turcotte R. On the need for bias correction in regional climate scenarios to assess climate change impacts on river runoff. Hydrology and Earth System Sciences, volumen 17 (número 3), 2013: 1189-1204.

Nash J.E. y Sutcliffe J.V. River flow forecasting through conceptual models part 1-A. Discussion of principles. Journal of Hydrology, volumen 10 (número 3), 1970: 282-290.

Peel M.C., Finlayson B.L., McMahon T.A. Updated world map of the Köppen-Geiger climate classification. Hydrology and Earth System Sciences, volumen 11, 2007: 1633-1644.

Rivas-Acosta I., Güitron de los Reyes A., Ballinas-González H.A. Vulnerabilidad hídrica global: Aguas Superficiales, en: Martínez P.F., Patiño C. (Ed.) Atlas de vulnerabilidad hídrica: Efectos del cambio climático en los recursos hídricos de México, Jiutepec, México, Instituto Mexicano de Tecnología del Agua, 2010, pp. 81-113.

Robles-Morua A., Che D., Mayer A.S., Vivoni E.R. Hydrological assessment of proposed reservoirs in the Sonora River Basin, Mexico, under historical and future climate scenarios. Hydrological Sciences Journal, volumen 60 (número 1), 2015: 50-66.

Seiller G. y Anctil F. Climate change impacts on the hydrologic regime of a canadian river: Comparing uncertainties arising from climate natural variability and lumped hydrological model structures. Hydrology and Earth System Sciences, volumen 18 (número 6), 2014: 2033-2047.

Tapia E.M., Minjarez I., Espinoza I. Use of Stella Software for the modelling of climate change impacts on water balance for the Rio Yaqui Basin, Sonora, Mexico. European Scientific Journal Edition, volumen 10 (número 14), 2014: 1857- 7431.

Teutschbein C. y Seibert J. Bias correction of regional climate model simulations for hydrological climate-change impact studies: Review and evaluation of different methods. Journal of Hydrology, volumen 456, 2012: 12-29. doi:10.1016/j.jhydrol.2012.05.052.

Troin M., Velázquez J.A., Caya D., Brissette F. Comparing statistical post-processing of regional and global climate scenarios for hydrological impacts assessment: A case study of two Canadian catchments. Journal of Hydrology, volumen 520, 2015: 268-288.

Velázquez J.A., Schmid J., Ricard S., Muerth M.J., Gauvin-St-Denis B., Minville M., Chaumont D., Caya D., Ludwig R., Turcotte R. An ensemble approach to assess hydrological models' contri- 
bution to uncertainties in the analysis of climate change impact on water resources, Hydrology and Earth System Sciences, volumen 17 (número 2), 2013: 565-578.

Velázquez J.A., Troin M., Caya D. Hydrological Modelling of the Tampaon River in the context of climate change. Tecnología y Ciencias del Agua, volumen 6 (número 5), 2015: 17-30.

Wilcoxon, F. Individual Comparisons by Ranking Methods. Biometrics Bulletin, volumen 1 (número 6), 1945: 80-83.

Wilks D.S. Statistical Methods in the Atmospheric Sciences, 2a ed., San Diego, Academic Press, 2006, 627 p.

\section{Citación sugerida:}

\section{Citación estilo Chicago}

Velázquez-Zapata, Juan Alberto, Magali Troin, Rodrigo Dávila-Ortiz. Evaluación del impacto del cambio climático en los indicadores hidrológicos de una cuenca del centro de México con base en un ensamble de modelos climáticos y en el modelo hidrológico SWAT. Ingeniería Investigación y Tecnología, XVIII, 03 (2017): 341-351.

\section{Citación estilo ISO 690}

Velázquez-Zapata J.A., Troin M., Dávila-Ortiz R. Evaluación del impacto del cambio climático en los indicadores hidrológicos de una cuenca del centro de México con base en un ensamble de modelos climáticos y en el modelo hidrológico SWAT. Ingeniería Investigación y Tecnología, volumen XVIII (número 3), julio-septiembre 2017: 341 351

\section{SemblanZas de los Autores}

Juan Alberto Velázquez-Zapata. Realizó sus estudios en ingeniería civil y la maestría en hidrosistemas (especialdad en ingeniería ambiental) por la Universidad Autónoma de San Luis Potosí. Obtuvo su doctorado en ingeniería civil en la Université Laval, en Quebec, Canadá. Desarrolló investigación postoctoral en la Université $d u$ Québec à Montréal, Canadá. Actualmente es investigador del CONACYT en El Colegio de San Luis. Sus intereses de investigación son la hidrología, la climatología, el impacto del cambio climático y los riesgos y desastres asociados a fenómenos hidrometeorológicos.

Magali Troin. Obtuvo el doctorado en geociencias ambientales por la Université AixMarseille (Francia). Desarrolló su investigación en modelación hidrológica y cambio climático durante su postdoctorado en la Université du Québec à Montréal (Canadá). Actualmente es investigadora en el Departamento de Ingeniería de la Construcción en la École de Technologie Supérieure (Canadá). Sus intereses de investigación son principalmente las relaciones entre el cambio climático y los recursos hídricos. Su enfoque consiste en la utilización de modelos higrológicos y agroecológicos como herramientas para la ayuda en la toma de decisiones y en la formulación de estrategias para la adaptación al cambio climático en los recursos hídricos.

Rodrigo Dávila-Ortiz. Se graduó de la Universidad Autónoma de San Luis Potosí en la carrera de ingeniería ambiental. Actualmente es becario de investigación en el programa agua y sociedad del Colegio de San Luis. Entre sus principales intereses de investigación se encuentran el estudio del impacto ambiental y el cambio climático. 